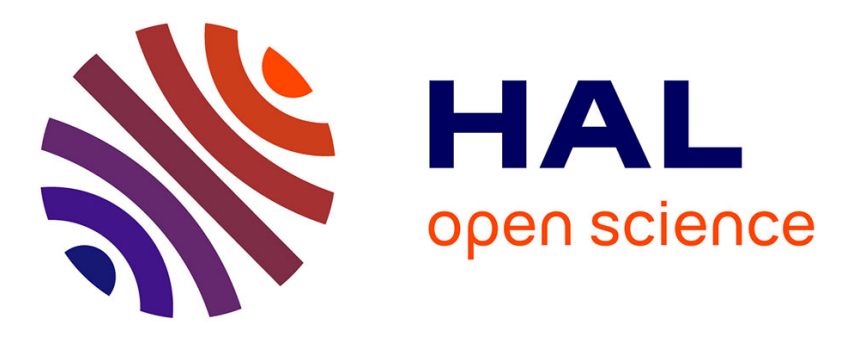

\title{
Le Mouvement moderne, le patrimoine et la figure des idéaux changeants
}

\author{
Jean-Baptiste Minnaert
}

\section{To cite this version:}

Jean-Baptiste Minnaert. Le Mouvement moderne, le patrimoine et la figure des idéaux changeants. Myriam Bacha. Architectures au Maghreb, XIXe-XXe siècles. Réinvention du patrimoine, Presses universitaires François-Rabelais; IRMC, Institut de recherche sur le Maghreb contemporain, pp.301314, 2011, Collection Perspectives Villes et territoires, 978-2-86906-260-3. halshs-01176273

\section{HAL Id: halshs-01176273 https://shs.hal.science/halshs-01176273}

Submitted on 17 Jul 2015

HAL is a multi-disciplinary open access archive for the deposit and dissemination of scientific research documents, whether they are published or not. The documents may come from teaching and research institutions in France or abroad, or from public or private research centers.
L'archive ouverte pluridisciplinaire HAL, est destinée au dépôt et à la diffusion de documents scientifiques de niveau recherche, publiés ou non, émanant des établissements d'enseignement et de recherche français ou étrangers, des laboratoires publics ou privés. 


\title{
LE MOUVEMENT MODERNE, LE PATRIMOINE ET LA FIGURE DES IDEAUX CHANGEANTS
}

\author{
Jean-Baptiste MINNAERT
}

Câest en 1965 que Peter Collins publie Changing Ideals in Modern Architecture, 1750-1950. Lârchitecte, critique et historien dôrchitecture anglo-canadien y entreprend dôxtraire lôhistoire de lôrchitecture moderne des lectures unificatrices et des téléologies mises en place dès les années 1930 par Sigfried Giedion (1941), Henry-Russell Hitchcock $(1932$; 1958) ou Nikolaus Pevsner (1936). Peter Collins leur préfère une approche plurielle et composite, qui restitue la diversité et lôncertitude des idéaux qui ont orienté les débats architecturaux et façonné leur histoire durant deux siècles de révolution industrielle. Ces idéaux changeants de lârchitecture des deux siècles précédents sont ainsi replacés dans une sorte de structure de malentendus ${ }^{1}$ sur laquelle repose lôhistoire qui en a été écrite depuis les années 1930 .

Il nântre pas dans nos intentions de détailler ici les riches et subtiles problématiques développées par Peter Collins, mais seulement dôtiliser la figure des idéaux changeants pour tenter de démontrer lâxistence dôın tournant idéologique qui, durant les années 1960 et 1970, aurait fait basculer le débat architectural et les politiques dôrrbanisme, de la suprématie accordée à une modernisation empreinte de machinisme, vers une attention accrue à la patrimonialisation de lârchitecture et des espaces urbains $^{2}$. Pour le dire autrement, la culture occidentale, au cours des années 1960, serait-elle passée dồn idéal prospectif à un idéal rétrospectif ? Aurait-elle vu le progressisme céder au culturalisme ${ }^{3}$ ? Doit-on parler dôın tournant patrimonial, ou plutôt dôın effet dôptique, dôıne abusive agrégation de phénomènes simplement convergents ?

La notion de patrimoine est aussi ancienne que les théories qui sont à lôrigine du Mouvement moderne; câest de plus fraîche date en revanche que la patrimonialisation concerne lộrchitecture et les espaces bâtis des $\mathrm{XIX}^{\mathrm{e}}$ et $\mathrm{XX}^{\mathrm{e}}$ siècles, qui, quantitativement, dominent largement les paysages urbains actuels. La patrimonialisation élargie à la période contemporaine est une évolution majeure dans lôhistoire du patrimoine. Sa topographie extensive lấrige de facto en chapitre à part entière des politiques dôırbanisme, lântroduit de plain pied dans lôhistoire de lâırbanisme de la période contemporaine et, ce faisant, ne peut quânduire des rétroactions avec lôhistoire de lârchitecture et de lâırbanisme du Mouvement moderne.

\footnotetext{
${ }^{1}$ En parlant de structure de malentendus, nous faisons allusion ici aux travaux de Harold Bloom (1973; 1975) qui, dans le domaine de la littérature, a théorisé sur le fait que toute lecture est déjà une interprétation, et que toute référence à un auteur devancier est une forme plus ou moins consciente de trahison.

${ }^{2}$ Notre propos repose sur lâxxtrapolation dôın "basculement patrimonial" (qui reste à démontrer) à partir des positions de Françoise Choay dans Lâallégorie du patrimoine (1992).

${ }^{3}$ Nous reprenons ici évidemment la paire de concepts utilisée par Françoise Choay, dans Urbanisme, utopies et réalités, une anthologie (1965). Le terme "culturaliste" désigne initialement un courant de lônthropologie américaine né dans les années 1930. Françoise Choay lômporte en histoire de lôrrbanisme, précisément durant les années 1960.
} 
À en croire lôhistorien, souvent facétieux, du postmodernisme Charles Jencks (1985), la mort du Mouvement moderne aurait été signifiée par le dynamitage de lậnsemble de logements sociaux de Pruitt Igoe à Saint Louis, Missouri (Minoru Yamasaki architecte), le 15 juillet 1972. Le tournant est évidemment plus composite. Les Congrès internationaux dôrrchitecture moderne (CIAM) sont dissous en 1959 ; la polémique du Pan-Am Building construit à New York en 1958-1963 par Walter Gropius voit non pas lâpothéose du maître du Bauhaus, mais son discrédit (M. L. Clausen, 2005). Les monstres sacrés du Mouvement moderne disparaissent les uns après les autres : Frank Lloyd Wright en 1959, Le Corbusier en $1965^{4}$, Walter Gropius et Ludwig Mies Van der Rohe en 1969. Sont publiés dômportants ouvrages critiques comme The Death and Life of Great American Cities de Jane Jacobs (1961), relectures théoriques comme Complexity and Contradiction in Architecture de Robert Venturi (1966) ou Teoria e storia dellâarchitettura de Manfredo Tafuri (1968), théorie de lârchitecture en milieu historique comme Lâarchitettura della Città dôAldo Rossi (1966). Ces ouvrages ont jalonné, chacun dans leur domaine, la fin de la phase étincelante dôın modernisme dorénavant taxé dôrrogance et dôutisme, voire dôacadémisme. Émergent au même moment de nouveaux référentiels, notamment ceux qui font de lấclectisme, intellectuel et formel, et du respect de la sédimentation historique des villes, les bases de nouvelles pratiques architecturales et urbanistiques, patrimoniales et historiennes.

$\mathrm{Si}$, au tournant des années 1960, le Mouvement moderne sấst effondré, tout au moins dans la définition qui était sienne depuis le début des années 1920, il sêst ensuite réinventé. On ne peut pas dire que les courants architecturaux qui en ont été les héritiers relèvent dône idéologie révolue. Lôrchitecture «héroïque et originale $»^{5}$ montre aujourdônui plus que jamais une belle vitalité, ne serait-ce que par lốlargissement des territoires sur lesquels elle sômplante. Durant le dernier tiers du $\mathrm{XX}^{\mathrm{e}}$ siècle et la première décennie du $\mathrm{XXI}^{\mathrm{e}}$, la modernisation nô cessé dô̂tre à lố uvre, lâaccélération du temps et des échanges est toujours plus vive. Depuis les années 1960, lândustrialisation des filières du bâtiment et des travaux publics ne cesse de sâccélérer. Cette industrialisation trouve, formellement, son hyperbole durant les années 1960 avec la naissance et lấpanouissement du métabolisme japonais, des Plug-in Cities ou Walking Cities dôArchigram, puis du courant High-tech. Souvent déterritorialisés, les projets conçus dans cette veine extrapolent ou rejouent les mythes modernistes, le poussant parfois à lôabsurde, au point dôen tirer souvent un élégant maniérisme ${ }^{6}$. Lôrchitecture progressiste, qui ne se limite assurément pas à cette veine, nô connu aucune éclipse technique, formelle ou conceptuelle. À lấchelle de lâırbanisme en revanche, des nuances sômposent. Sans parler de la forme «tours et barres» des grands ensembles, rapidement vilipendée, les mégastructures, les structures proliférantes ou les nappes, élaborées et pratiquées durant les années 1950 à 1970, notamment dans la suite des réflexions de Team X (M. Risselada, D. van den Heuvel, 2005), ont abouti à des formes très audacieuses et souvent subtiles, mais ont quelques fois peiné à répondre aux besoins culturels, sociaux, politiques et économiques de requalification des centres dégradés des villes occidentales et de réinvention de lârrbanisation de leurs périphéries. Les décideurs politiques leur ont parfois préféré des formes alternatives où se renouent, à un titre ou à un autre, des rapports à la mémoire, à lôhistoire, voire au patrimoine.

Au milieu des années 1960 émerge le postmodernisme qui a fortement imprimé sa marque dans la production architecturale et urbanistique des années 1970-1980. Sâst plus durablement imposé ce quôon appelle aujourdôhui le New Urbanism, mouvement consacré aux États-Unis au début des années 1980, mais qui prolonge les théories élaborées et les projets réalisés en Europe dès les années 1960 par des figures comme François Spoerry, Léon Krier, Maurice Culot, ainsi que Demetri Porphyrios ou Quinlan Terry, sans omettre le rôle théorique majeur de Manfredo Tafuri $(1979 ; 1982)$

\footnotetext{
${ }^{4}$ Dans son ouvrage Architecture en France, 1940-2000, histoire et théories, Jacques Lucan (2001) montre bien en quoi "lâffondrement de la statue du Commandeur" a désinhibé le débat architectural français, avant même la crise de Mai 68.

${ }^{5}$ Le terme, devenu célèbre, est de Robert Venturi.

${ }^{6}$ Le High-tech au demeurant a ouvert la voie à un nouvel idéal techniciste, que recensa et promut lâxxposition Architectures non standard au Centre Georges Pompidou en 2003 (F. Migayrou, Z. Mennan, 2003). Voir aussi Antoine Picon (2010).

${ }^{7}$ Le courant du New Urbanism, né dans le même contexte que le postmodernisme, lui est souvent assimilé, alors que, détails discriminants, il ne pratique pas lôronie critique chère aux postmodernes et prend des positions urbanistiques beaucoup plus radicales à lâncontre de la Charte dôAthènes.
} 
et lâmpact médiatique des prises de position du Prince Charles et de son école ${ }^{8}$. Largement pratiqué aujourdôhui de par le monde, revendiquant aussi le qualificatif plus ancien de néo-traditionnel, parfois appelé mouvement de Renaissance urbaine, le New Urbanism réhabilite la syntaxe et la composition classiques ou vernaculaires, renoue avec la rue et les places. Il puise ses échelles spatiales et ses solutions formelles à lôhistoire de lôarchitecture et de la ville, ainsi quấ nombre dôpérations de réhabilitation patrimoniale, pour les appliquer à des projets dôrbanisme où, pour le dire vite, lấchelle du piéton et de la rue à échoppes prime dorénavant sur celle de lâutomobile et des $7 \mathrm{~V}$ corbuséennes, et où lốclectisme classicisant des volumes et des façades périme les allégories machinistes du Mouvement moderne. Le New Urbanism, enfin, produit de la ville ramassée imitant la densité des centres-villes historiques, même lorsque les projets sont implantés en situation suburbaine, périurbaine ou balnéaire, au contraire des Modernes qui, sôntéressant aux centres urbains, en préconisaient le desserrement sans dôilleurs toujours le pratiquer.

Lôhistorien dôujourdôhui constate les faveurs plus systématiques que, depuis les années 1960, les élites politiques et culturelles, les urbanistes et les architectes, les maîtres dôuvrage et le public accordent aux projets qui recyclent ou au moins respectent la "tradition" et le "patrimoine"". Cependant, on ne peut opposer frontalement le Mouvement moderne à ces pratiques culturalistes et à ces architectures néo-traditionnelles, ne serait-ce que parce que lôın et les autres sont avant tout projets et relèvent dôdéologies à visées prospectives. A contrario, les Modernes eux-mêmes ont qualifié leur mouvement de nouvelle tradition ${ }^{10}$.

Ces idéaux changeants ont des contours et des statuts, sémantiques, culturels, historiques, difficilement comparables terme à terme. Il faudrait dôailleurs commencer par identifier les espaces et les lieux où sôppliquerait ce basculement patrimonial. En raison de la croissance même des villes, les objets architecturaux et urbains patrimonialisés sont majoritairement centraux, ou le sont devenus, ce qui leur offre une position culturelle avantageuse et surdétermine peut-être leur importance par rapport à la quantité globale de gestes architecturaux et urbains en lesquels nous vivons aujourdôui. Si lônn se place au centre dône ville européenne, le tournant patrimonial des années 1960 est dôine évidence éclatante. Si lôn appréhende le phénomène depuis des espaces non urbanisés ou périurbains, ou bien à lấchelle des réseaux routiers, ferroviaires ou aériens continentaux ou transcontinentaux, ce tournant est plus difficile à percevoir. À moins que ces objets patrimoniaux, par lômportance culturelle ou touristique que leur ont conférée les sociétés qui les ont fabriqués et celles qui les réinventent aujourdôhui, ne constituent précisément une maille des dits réseaux.

Il serait absurde dôffirmer que lâdéal patrimonial, tel un bernard-lârmite, aurait remplacé lâdéal moderniste en ses lieux et places, géographiques et intellectuels, dans le courant des années 1960. Les moments du basculement sont aussi composites que ses lieux. Les notions dôrchitecture mineure et de patrimoine urbain trouvent droit de cité patrimonial dès la Conférence dôAthènes de 1931 (2002), et entrent dans le champ législatif français, avec les périmètres de protection de 500 mètres prévus par la loi de 1943, et surtout avec la loi Malraux de 1962 sur les secteurs sauvegardés ${ }^{11}$. La reconstruction de

\footnotetext{
${ }^{8}$ Après des réalisations phares comme Port-Grimaud sur la Côte dôAzur dès les années 1960 (François Spoerry architecte), puis Poundbury en Grande Bretagne à la fin des années 1980 (Léon Krier architecte), Célébration près de Walt Disney World Resort en Floride au début des années 1990, ou encore Val dâEurope près de Disneyland Paris à la fin des années 1990, de récentes réalisations montrent toute la vitalité du mouvement : on peut citer le très récent village dốl Gouna en Égypte sur la Mer Rouge, et les nouveaux quartiers du PlessisRobinson, dans la banlieue de Paris, de Xavier Bohl et Marc et Nada Breitman, étudiés initialement avec François Spoerry. Cette dernière réalisation a reçu en 2008 le Prix européen dôrchitecture Philippe Rotthier. La Déclaration de Bruxelles (1978) constitue lâın des textes de ralliement de lâırbanisme néo-traditionnel, ce New Urbanism européen. Voir : (M. Culot, P. Lefèbvre, 1980). Le Congress for New Urbanism, fondé en 1993 aux États-Unis, a publié en 1996 une Charte de lâırbanisme. www.cnu.org. Voir Peter Katz (1994). Voir aussi Cynthia Ghorra-Gobin (2006). http://www.cdu.urbanisme.equipement.gouv.fr/IMG/pdf/newurbanism_ cle65d7e2.pdf

${ }^{9}$ Laissons pour le moment ces termes à leurs guillemets, pour restituer lâmprécision sémantique de leur usage courant.

10 Nous ne faisons pas nécessairement référence ici à lôouvrage dôEric Hobsbawm et Terence Ranger, Lônvention de la tradition, (2006), mais à lôuvrage de Sigfried Giedion, Space, Time and Architecture (1941).

${ }^{11}$ Les paysages ont pu être protégés en France dès la loi de 1906 puis grâce à celle de 1930 (qui font écho aux lois de 1887 et, surtout, de 1913 sur les Monuments historiques).
} 
lấlot insalubre n ${ }^{\circ} 16$ à Paris à partir de 1943 (S. Texier, 2005) par sa sensibilité au bâti ancien et grâce à lêxpérimentation des pratiques de réhabilitation, contribua à préfigurer les restaurations de centres anciens, en particulier les secteurs sauvegardés qui entrent en vigueur à partir des années 1960.

Alors que la doctrine du postmodernisme et celle du New Urbanism ont dôbord été définies dans le cadre de projets dôurbanisme et de logement, lâne des principales motivations à la lente construction juridique du patrimoine architectural et urbain relève du développement du tourisme, dont les rapports avec le patrimoine sont consubstantiels depuis plus de deux siècles (C. BerthoLavenir, 1999 ; C. Zytnicki, H. Kazdaghali, 2009). Les protagonistes de la symbiose entre tourisme et patrimoine furent dès la fin du XIX ${ }^{\mathrm{e}}$ siècle en France le Touring Club de France (M. Bacha, 2009), lôAutomobile Club de France ou le Club Alpin Français, notamment. Parallèlement à leur action concernant le développement des infrastructures routières et hôtelières, ces organisations ne contribuèrent pas peu à étendre à lôménagement du territoire et à lâsppace urbain le concept de pittoresque, ainsi que le rapport à la mémoire et à lôhistoire.

Le tourisme est un outil de structuration du territoire si puissant, que là où le monument et le paysage font défaut, ils seront inventés ${ }^{12}$. Sôl nôsst plus guère iconoclaste dôfffirmer que le patrimoine est devenu aujourdônui une dimension parmi dôutres des politiques dôırbanisme et de valorisation des territoires, le tourisme a été et demeure un puissant vecteur de ce phénomène. Serait-il provocateur dôffirmer que le commerce et le développement économique constituent le but du tourisme; les infrastructures routières, ferroviaires, aériennes et hôtelières en sont les moyens, et les paysages et monuments architecturaux et urbains les prétextes ? Cela expliquerait lâctuelle fortune, collatérale pour ainsi dire, du New Urbanism. En effet, celui-ci peut être vu, dôıne certaine manière, comme lấquivalent à neuf et hors les murs de la réhabilitation patrimoniale des centres anciens. Les cas français de Port-Grimaud ou Val dôEurope près de Disneyland Paris, constituent à ce titre des exemples éloquents, que conforte a contrario le fait que Marc Augé ait parlé de disneylandisation des centres anciens patrimonialisés (M. Augé, 1997). Comme le remarque en effet lânthropologue, les parcs à thème, notamment ceux de Disney, peuvent être lus comme du patrimoine hors-sol et horstemps. Là où les aménageurs et les acteurs économiques estiment quôl est le plus rentable de le faire, ces parcs à thème fournissent tout, voire davantage, que ce que les touristes attendent dône destination "authentique" sur le plan patrimonial. Entre Disney et les villes historiques, la concurrence commerciale est dôutant plus rude quâl est plus rapide de construire un parc à thème à proximité dôıne ligne TGV ou dôın aéroport (comme cốst le cas avec Disneyland Paris), que de faire venir le TGV à une ville-patrimoine. De ce point de vue, les parcs à thème, avec leur architecture savamment factice, sont sans doute à lire comme une forme nouvelle de ce balnéaire que les historiens ont érigé en objet dấtude depuis trente ans.

Tout cela nômènerait-il pas à conclure sans autre forme de procès que le patrimoine nâst finalement quânn saut qualitatif opéré par des acteurs économiques qui, par le canal du tourisme et accessoirement par celui du tourisme patrimonial et culturel, visent invariablement à servir et asseoir la suprématie que ce quôon appelle aujourdôhui le shopping ${ }^{13}$ exerce depuis longtemps sur les espaces urbains et les paysages, quel que soit leur implantation par rapport aux centralités et quelles que soient les formes, "modernes" ou "traditionnelles", qui leur sont imprimées ? Pour le dire autrement, le patrimoine ne succéderait pas au Mouvement moderne en tant quôdéologie dominante, mais ne serait quânn phénomène de la modernisation.

Maintenons malgré tout lôhypothèse que lâdéal du Mouvement moderne et lâdéal patrimonial se succèdent, même si ceux-ci ne sânchaînent pas exactement, ne sôpposent pas terme à terme et

\footnotetext{
${ }^{12}$ À plusieurs reprises, dans les débats auxquels nous avons assisté dans le cadre du programme de recherche dirigé par Myriam Bacha à lâRMC Tunis, est revenu le terme de "bidonnage patrimonial", notamment à propos du cas de lânvention patrimoniale du village touristique de Sidi Bou Saïd à partir de 1915. Voir Hamon (infra) et également les recherches en cours, et à paraître, de Charlotte Jelidi.

${ }^{13}$ Voir le shopping que Rem Koolhaas analyse dans: Harvard project on the cityé (2000); Voir aussi Alain Bourdin (1992). Dans son exposition Cronocaos à la Biennale dôrchitecture de Venise 2010, Rem Koolhaas est allé plus loin encore dans la possible merchandisation de la notion de patrimoine. Partant du constat que $22 \%$ des terres émergées de la planète sont dorénavant soumises à un régime de protection quelconque, il propose une sortie de la sacralisation du patrimoine en proposant, par exemple, la mise en place dôn marché global du patrimoine, calqué sur le modèle du marché international des droits dấmission du CO2. Voir lậntretien de Rem Koolhaas avec Frédéric Edelmann, Le Monde, 5 septembre 2010 ; voir aussi Sejima (2010).
} 
fonctionnent sur des registres politiques et culturels différents. Car, enfin, les acteurs qui ont appliqué ces idéaux successifs sont bel et bien passés de lồn à lâuutre, lors dốvénements précis ${ }^{14}$. Si nous poursuivons notre hypothèse de départ, postulons que la phase étincelante de lâdéal patrimonial commencerait dans les années 1960, évidemment avec la Charte de Venise (1964), puis la convention de lốnesco sur le patrimoine mondial (1972). En France, le phénomène sôgrègerait dès la loi Malraux sur les Secteurs sauvegardés (1962), puis autour du classement ou de lônscription dồn nombre important dôrchitectures du XIX ${ }^{\mathrm{e}}$ et du $\mathrm{XX}^{\mathrm{e}}$ siècles à partir de 1974. Ces classements sont pour bonne part consécutifs à la démolition des Halles de Baltard en 1971-1973 ${ }^{15}$, qui souleva un scandale convoquant lôpinion publique à une échelle inédite dans la France dôlors. Il est vrai que cette opinion avait déjà été échaudée par lốchèvement en 1967 de la voie expresse rive droite, construite sur une berge de la Seine, en vis-à-vis dôhôtels particuliers du XVII ${ }^{\mathrm{e}}$ siècle se déroulant sur la berge opposée, celle de lậle Saint-Louis et de lậle de la Cité, et qui seront intégrés plus tard dans un périmètre classé au patrimoine mondial. De même, les excès de la politique volontariste de construction de grands ensembles entamée en 1950, sont critiqués dès les années 1960, non seulement par les sociologues et les philosophes (H. Lefebvre, 1968), mais surtout par les architectes et les urbanistes eux-mêmes. La circulaire Guichard (1973) imposera progressivement à la technocratie française et au secteur du bâtiment et des travaux publics de mettre un terme à la construction de ces grands ensembles, et dômaginer, pour le logement social en particulier, des formes architecturales moins denses. La crise de Mai 1968 catalyse une volonté dônggiornamento culturel tous azimuts. Elle bouleverse la culture professionnelle des architectes et lậnseignement de lârchitecture qui, après lốclatement de lớcole des Beaux-arts, sôuvre aux sciences sociales et à lốistoire en particulier. Le choc pétrolier de 1973 et la crise économique qui sâensuit précipitent le déclin de lândustrie lourde européenne, suscitent un désenchantement vis-à-vis du machinisme et garantissent le succès de la notion de patrimoine industriel, née en Grande-Bretagne dès les années 1940. Ces événements tels que présentés ici, plaident a priori pour lôhypothèse du basculement qui, en moins de quinze ans, vit la fin de la toute-puissance de lâdéal moderniste et la montée en puissance de lâdéal patrimonial.

Cet idéal patrimonial, de surcroît, influe sur les pratiques urbanistiques déjà établies, et notamment sur lônterprétation de règlements urbains qui nốtaient pas initialement conçus pour lui faire place. En France, les années 1970 voient lânstruction des Plans dôccupation des sols (POS) et la mise en î uvre des Zones dôménagement concertées (ZAC), deux outils créés dans le cadre de la Loi dôrientation foncière de 1967. Alors que le POS a été conçu par les législateurs essentiellement comme un plan de zonage, celui de Paris qui est mis en application en 1977 prescrit, notamment, un retour partiel du bâti sur rue à des volumétries post-haussmanniennes. Avec le temps, les POS vont inclure de plus en plus de dispositions concernant la qualité du paysage urbain, voire le patrimoine architectural. Si les ZAC sont des dispositifs où domine initialement la pratique de la démolition-reconstruction, les formes bâties qui sont produites dans ce cadre affirment progressivement leur sympathie pour la ville traditionnelle, réinterprétée avec une inventivité et une finesse croissantes, tout en promouvant la créativité des architectes contemporains. Après les Secteurs sauvegardés, et avant les Zones de protection du patrimoine architectural et urbain (ZPPAU, créées en 1983; devenues en 1991 ZPPAUP, le P final pour "paysager"), deux dispositifs spécifiquement patrimoniaux, les POS sont lồn des vecteurs du mouvement de contrôle qualitatif architectural des droits à construire dans les centresvilles. Les POS opèrent à une échelle plus vaste que les secteurs sauvegardés et les ZPPAUP, et surtout imposent par la règle une logique quantitative qui banalise la pratique de formes architecturales et urbaines dialoguant avec lôhistoire ${ }^{16}$.

\footnotetext{
${ }^{14}$ Il en va ainsi du maire de Tours, Jean Royer dont lốlection en 1959 cö̈ncide avec lônterruption de lôpération du secteur de rénovation en cours pour ce qui concerne la partie la plus patrimoniale du vieux Tours, pour laisser à lôrchitecte Pierre Boille qui rénove depuis 1956 lâhôtel des Compagnons du Devoir puis lổlot qui lântoure, lônitiative de sensibiliser Pierre Sudreau (ministre de la Construction) à une pratique qui sera très bientôt celle des Périmètres de restauration immobilière (PRI) qui, eux-mêmes, annoncent les plans de sauvegarde et de mise en valeur des Secteurs sauvegardés institués par la loi Malraux de 1962, voir Nelly Alletru (2007).

${ }^{15}$ La destruction de la gare dôEuston à Londres en 1962 souleva en Grande-Bretagne une polémique comparable.

${ }^{16}$ Avec la loi Solidarité Renouvellement Urbain (SRU) du 13 décembre 2000, les POS ont été remplacés par les Plans locaux dôrrbanisme (PLU). Avec la loi Grenelle 2 sur lậnvironnement du 10 juillet 2010, les ZPPAUP laissent place aux Aires de mise en valeur de lôrchitecture et du patrimoine (AVAP).
} 
Quôls soient ou non spécifiquement patrimoniaux, ces nouveaux documents dôirbanisme ont été des leviers de "reconquête" - traduire gentryfication - des villes-centres, par le moyen de la réhabilitation, ou par celui de la construction en continuité esthétique, morphologique ou fonctionnelle avec lâxistant, alors que les modernes au contraire voyaient les centres-villes comme irrémédiablement déclassés et nécrosés et les vouaient à une destruction-reconstruction zonée, à effectuer par-dessus une voirie redessinée et calibrée, après abolition ou refonte du parcellaire. Cette profonde révision des représentations et des modalités dôntervention sur les villes et leurs centres constitue, en France, le fertile terreau de lâdéal patrimonial.

Cependant, la figure des idéaux changeants de Peter Collins ne semble transposable au cas que constitue le supposé passage dône idéologie progressiste à une idéologie culturaliste, quânec de grandes précautions de méthode. Pour ce faire, il faut commencer par reprendre le sens que Collins, travaillant à lôntérieur des corpus architecturaux qui ont fondé le Mouvement moderne, a donné à cette notion dôdéaux changeants. Opposer lôdéal moderniste à lôdéal patrimonial suppose, notamment, de pouvoir rabattre ce dernier à sa dimension de pratique architecturale et urbanistique, de manière à le rendre plus comparable à ce qui relève des pratiques et représentations modernistes, essentiellement projectuelles. En soi, lâxercice est roboratif, dans la mesure où lôhistorien doit alors appréhender ce qui, par exemple, dans une intervention sur le bâti ancien, relève non pas nécessairement de la préservation, mais de lônvention. Même ramenée à cette dimension, on ne peut toujours pas considérer quâl y ait un passage de lâdéologie moderne à lâdéologie patrimoniale. Les architectes des Monuments historiques, par exemple, nônt évidemment pas attendu la fin du Mouvement moderne pour pratiquer le projet architectural dôentretien et de restauration des édifices classés. Câsst dôailleurs lấthique et la méthode de restauration que Viollet-le-Duc développa au contact des monuments médiévaux qui fonda le rationalisme structurel dont se réclameront ensuite les Modernes. Quant à la notion de patrimoine urbain, elle émerge, on lâ vu, dans les mêmes décennies que la formulation théorique de lârrbanisme moderne. Ni à lấchelle du projet architectural, ni à celle du projet urbain, on ne peut donc parler de succession, dans la mesure où ces idéaux, moderne dône part, patrimonial de lâutre, ne sâppliquent pas aux mêmes objets, ni toujours aux mêmes échelles : espaces à construire dồn côté, bâti existant à réinvestir de lôuutre. Dâutre part, nombre dôdéaux de lôrchitecture moderne fonctionnent sur le mode de lânalogie, voire de la métaphore, dimensions qui ne sont pas si simples à déceler dans les représentations et les pratiques patrimoniales. Par ailleurs, les architectes et maîtres dôuvrage qui pratiquent la construction et ceux qui î uvrent en réhabilitation, restauration et valorisation touristique ne sont pas toujours les mêmes. Le seraient-ils, les contextes techniques, économiques, réglementaires, urbanistiques et politiques de leur action diffèrent en grande partie.

Si lôn constate globalement un tournant patrimonial à partir des années 1960, il est à situer sur le registre large de lôhistoire culturelle, plus que sur celui de lôhistoire de lôrchitecture et de lâırbanisme proprement dit. Plutôt que dômaginer quôin idéal succède à un autre, il est plus réaliste de concevoir que ces modèles et les mentalités qui les véhiculent se superposent et sâentremêlent, ce que Peter Collins au demeurant démontrait dans Changing Ideals. Ces idéaux ne se stratifient pas, mais sôrticulent en des combinaisons temporelles, géographiques et culturelles complexes, toujours singulières et irréductibles dồn cas à un autre. Ces combinaisons sont faites de coexistences, interactions et superpositions décalées.

Les ruptures quôon a pu identifier plus haut relèvent dốvénements placés en séries par lôhistorien. Ce sont des événements signifiants, mais parfois surdéterminés, qui prennent en leur temps, ou rétrospectivement, une dimension fondatrice. Avant que ne bascule la configuration des objets architecturaux et urbains, ont dôabord basculé lômaginaire et lôdéologie des acteurs, notamment les historiens. Le public, les élites politiques, les critiques, les historiens ont rêvé de la modernité puis sân sont désenchantés. Cela ne signifie pas que cette modernité à lô̂ uvre se soit interrompue faute dô̂tre dorénavant rêvée aussi intensément que par le passé. Ces divers acteurs ont réévalué le rôle de la mémoire et du patrimoine, construisant de nouvelles lectures ï et peut-être de nouvelles téléologies ï qui, dans un second temps, ont influé partiellement sur la production des espaces et du bâti. 
Pour mieux comprendre le problème de méthode que soulève cette soi-disant succession dôdéaux, il nôst pas inutile de plonger un peu dans la théorie de lôhistoire ${ }^{17}$. Ainsi pourrait-on dépasser les lectures traditionnelles qui, explicitement ou implicitement, ont encore largement cours en histoire de lôrt : croyance en des influences unilatérales exercées par des émetteurs actifs sur des récepteurs passifs, surdétermination de lârtiste ou du décideur érigés en prophètes de la réalité, limitation de lôhistoire de lârchitecture à une histoire des vanités et de lânnovation, survalorisation de lâxxceptionnel monumental par rapport au banal sédimenté, et de lâurbanisme par rapport à lôırbanisation, etc. De nombreux modèles interprétatifs ont été importés en histoire de lôrchitecture, en particulier par le canal des aires ex-coloniales. Ces terrains foncièrement difficiles sur le plan méthodologique ont imposé des innovations méthodologiques qui, désormais, profitent à tout le champ disciplinaire.

Par exemple, Mercedes Volait, Joe Nasr (2003) et Anthony King (2000) et nous-même (J.B. Minnaert, 2005), avons pratiqué la figure de la circulation des modèles dans une géométrie multilatérale, qui restitue lômmense variété des situations et des trajectoires individuelles, collectives, praticiennes et intellectuelles ${ }^{18}$. En dépit de la fécondité dồne telle méthode, se limiter à ne pister que les modèles (modèles formels et fonctionnels des architectes et des urbanistes, ou modèles interprétatifs des historiens) et leurs applications et interprétations locales, reste encore une manière descendante ou centrifuge dốcrire lâhistoire : un modèle sâxpérimente, sấnonce, puis se diffuse et sôpplique, ou non, à des terrains où, éventuellement, ils sont transformés, hybridés, diffusés, voire refusés. Cette méthode sêst affinée en ne sôntéressant plus seulement aux modèles, mais aussi aux hommes qui, éventuellement, les élaborent, transmettent, appliquent, transforment ou refusent. Ici lôpproche à échelles multiples, notamment micro-historique (B. Lepetit, 1996, 71-93) et prosopographique prend toute son importance.

Dans toutes ces configurations, la succession des idéaux, modernistes puis patrimoniaux, est fortement affinée ou relativisée par la priorité que donne leur historien aux aspérités de leurs changements de rapports, et aux modalités concrètes de leur circulation (par quels canaux institutionnels, par quels hommes ?). Lôhistoire tend ici à être vue aussi dôen bas, les archives locales jouant alors un rôle fondamental.

Afin de mieux construire une réflexion sur la figure des idéaux changeants qui éclairerait le soidisant passage de lôdéal moderne à lâdéal patrimonial, il serait également intéressant de puiser à dôutres modèles théoriques ${ }^{19}$, notamment à la théorie des transferts culturels élaborée par Michael Werner et Michel Espagne (1999) et surtout à la théorie de lôhistoire croisée du même Michael Werner (2003, 7-36), à laquelle nous limiterons ici nos réflexions.

Fondamentalement multidimensionnelle, lôhistoire croisée est aisément transposable en histoire de lôrchitecture et des formes urbaines, coloniale en particulier, et ouvre aux historiens de ces champs des perspectives très fécondes ${ }^{20}$. Michael Werner $(2003,30)$ insiste sur le fait quôl est important de

\footnotetext{
${ }^{17}$ Exercice auquel nous nous sommes livrés dans un numéro sous notre direction de la revue Histoire de lâart (2006), ainsi que dans un article, "Architecture ordinaire et hommes pluriels" (2009).

${ }^{18}$ On pourrait ajouter à cette figure une structure multi-temporelle : parallélisme des pratiques dône époque à lôutre, superpositions et interactions des durées, etc.

${ }^{19}$ Ainsi la théorie de la réception de Hans Robert Jauss et Wolfgang Iser (H. R. Jauss, 1978, 89-134) ; la théorie des systèmes dôcteurs de Michel Crozier et Erhard Friedberg (1977); la théorie de lârchéologie générale de Philippe Bruneau. Dérivée de la théorie de la médiation de Jean Gagnepain, et assez peu connue dans le champ de lâhistoire de lârchitecture contemporaine, la théorie de lârchéologie générale fut exposée, souvent de manière délicieusement provocatrice, par Philippe Bruneau et Pierre-Yves Balut, principalement, dans les multiples numéros de la revue Ramage, ainsi que dans Philippe Bruneau, Pierre-Yves Balut (1997).

${ }^{20}$ «Sur un plan intellectuel, le áournant culturalisteâ en mettant lộccent sur la spécificité, voire le caractère irréductible du local, a contribué à affiner les connaissances sur le fonctionnement différencié des sociétés et des cultures, tout en entraînant une fragmentation des savoirs et, par là même, leur relativisation. Les interrogations consécutives à lâfffondrement du colonialisme ont, par ailleurs, affecté la position jusquâalors dominante des sciences sociales óccidentalesô Désormais soupçonnées dômpérialisme intellectuel et de stratégies de domination politique, elles voient leur ambition universaliste affaiblie. [é ] Mais lâhistoire croisée ambitionne aussi de traiter dôbjets et de problématiques spécifiques qui échappent aux méthodologies comparatives et aux études de transferts. Elle permet ainsi dôppréhender des phénomènes inédits à partir de cadres dônalyse renouvelés. Ce faisant, elle fournit lôccasion de sonder, par un biais particulier, des questions générales telles
} 
garder à lâsprit que lôhistoire croisée se pratique nécessairement à la double échelle des faits et des questionnements auxquels ceux-ci sont soumis. Lôhistorien qui écrit les faits est lui-même pris dans lôhistoire ; il ne neutralisera jamais entièrement ses propres positionnements culturels, ni les lignes et les impensés de sa propre biographie. Il inscrit des faits dans les temps particuliers à son enquête et à ses méthodes. Il nốchappe jamais totalement aux téléologies de son époque, ni à celles du passé de sa discipline. Lôhistorien en effet travaille sur la base dône historiographie qui est une longue sédimentation historique, faite à la fois de traditions, de déchirures, de réinventions et de rémanences. Lôhistorien invente ses objets par les méthodes et par les échelles de temps et dôespace avec lesquelles il les approche. Les faits quâl observe et décrit entretiennent avec lui, et entre eux, des relations fluctuantes. La méthode de lôhistoire croisée est donc toute dôjustements et dôncessante réflexion sur les conséquences de ses propres choix de méthode.

Les temps de lôhistoire sont à la fois différentiels et imbriqués. Il nô a pas dôhorloge extérieure qui alignerait au même pas les objets, les méthodes qui permettent de les définir et analyser, et les historiographies dans lesquels ils sônscrivent. Lôhistoire croisée vise à dépasser lôpposition entre la synchronie que lôn reproche à lôhistoire comparée, et la diachronie à laquelle sont souvent réduits les transferts culturels.

Relier lônvention, le maniement, le vécu et les représentations du patrimoine aux modalités de lôcte collectif de bâtir, est une manière plus efficace encore de démasquer les préjugés idéologiques dont lâhistoriographie de lôhistoire de lôrt peut parfois apparaître comme une longue succession, de surcroît oublieuse dôllle-même.

Ce supposé passage de lâdéal moderniste à lâdéal patrimonial ne relève pas premièrement dônn basculement des pratiques architecturales et urbaines en elles-mêmes, côest à dire du passage dône modernisation à outrance à une patrimonialisation à outrance, bien quâl y ait eu des évolutions en ce sens et surtout une construction historienne de faits qui tende à le laisser penser. Ce basculement relève dône modification de représentation culturelle que lôpinion dominante en son temps puis celle des générations suivantes, dôıne part, et les historiens dôlors puis ceux des générations suivantes dôutre part, ont socialement et intellectuellement construite. Le phénomène relève dôabord du simple contrepied: à la violence technocratique de la modernisation des Trente Glorieuses, le critique, lôhistorien et leurs lecteurs se sont imposés le devoir dôpposer la raison culturaliste, démocratique, dồn rapport maîtrisé au passé et à la mémoire par la construction de figures patrimoniales diverses. $\mathrm{Au}$ temps court par lequel Le Corbusier pensait son lien aux traditions, antique, méditerranéenne, classique, rationaliste, les théoriciens-architectes et les historiens «culturalistes » répondent par le temps long conçu jadis par les protagonistes de lấcole des Annales, mais aussi, dôıne certaine manière par le sociologue Maurice Halbwachs. Au demeurant, la fertile dialectique progressisme-culturalisme que Françoise Choay introduisit en 1965 dans Urbanisme, utopies et réalités fixa durablement les termes du débat que nous explorons.

Les questions abordées ici relevaient naguère de la critique autant que de la passion ï ce qui nâst guère évitable avec le patrimoine. Elles peuvent aujourdôhui entrer de plain pied dans le champ, espérons-le raisonné, de lôhistoire. Lôistoricisation des faits nêst pas simplement possible parce que la péremption de ceux-ci les inscrirait à distance raisonnable. Certains outils théoriques facilitent le passage des faits du stade de la critique à celui de lôhistoire, du registre de lậngagement citoyen ou esthétique à celui de leur mise à distance historiographique, indépendamment de leur position dans le temps. Le passage dônn idéal moderniste à un idéal patrimonial est sans doute lâne des problématiques par lesquelles une histoire croisée peut sâxprimer de manière féconde en histoire de lộrchitecture. Nous avons tenté ici dộn appliquer quelques principes.

En introduction à ses Douze leçons sur lôhistoire, Antoine Prost $(1996,9)$ rappelle que la plupart des historiens «se dispensent, au début de leurs livres, de définir les concepts et les schémas

que celle des échelles, des catégories dônalyse, du rapport entre diachronie et synchronie, des régimes dôhistoricité et de la réflexivité. Enfin, elle pose le problème de sa propre historicité à partir dône triple procédure dôhistoricisation : de lôbjet, des catégories dônalyse et des rapports entre le chercheur et lôbjet. Elle offre ainsi une óboîte à outilsôqui, par-delà les sciences historiques, peut être opérationnelle dans de nombreuses autres disciplines qui croisent des perspectives du passé et du présent » (M. Werner, B. Zimmermann, 2003, 710). Dans cet important article, les auteurs établissent des liens avec les Connected History, Shared History et Entangled History anglo-saxonnes. 
dônterprétation quâls mettent en î uvre, quand leurs collègues allemands sôg sentiraient tenus. À plus forte raison, ils estiment prétentieux et périlleux dêentreprendre une réflexion systématique sur leur discipline : ce serait revendiquer une position de chef dốcole qui répugne à leur modestie ï fût-elle feinte $\ddot{i}$ et qui, surtout, les exposerait à la critique peu bienveillante de collègues auxquels ils craindraient de paraître vouloir apprendre leur métier. La réflexion épistémologique semble porter atteinte à lấgalité des ómaîtresô de la corporation. Sân dispenser est éviter à la fois de perdre son temps et de sôfffrir aux critiques des pairs. Heureusement, cette attitude est en train de changer ».

\section{BIBLIOGRAPHIE}

AlLETRU Nelly, 2007, La sauvegarde du Vieux Tours sans secteur sauvegardé : création et mise en î uvre dôın périmètre de restauration immobilière de 1961 à 1973, mémoire de master 2, Université de Tours François-Rabelais, sous la direction de Jean-Baptiste Minnaert, 2 vol.

Auge Marc, 1997, Lốmpossible voyage, Payot, Paris.

BACHA Myriam, 2009, "Patrimoine et tourisme en Tunisie au début du Protectorat : interactions et dépendances", in Colette Zytnicki et Habib Kazdaghli (dir.), Le tourisme de lâEmpire français. Politiques, pratiques et imaginaires (XIXe-XXe siècles) : un outil de la domination coloniale?, Publications de la Société française dồistoire de lốutre-mer, Saint-Denis, 2009, 155-163.

Bertho-LaVenir Catherine, 1999, La roue et le stylo. Comment nous sommes devenus touristes, Odile Jacob, Paris.

BLoom Harold, 1973, The Anxiety of Influence. A Theory of Poetry, Oxford University Press, Oxford (seconde édition 1997).

BLoom Harold, 1975, A Map of Misreading, Oxford University Press, Oxford.

BouRdin Alain, 1992, "Patrimoine et demande sociale", in Régis Neyret (dir.), Le patrimoine, atout du développement, Presses universitaires de Lyon, Lyon.

Bruneau Philippe et Balut Pierre-Yves, 1997, Artistique et archéologie, Presses de lâıniversité ParisSorbonne, Paris.

CHOAY Françoise, 1965, Urbanisme, utopies et réalités, une anthologie, Le Seuil, Paris.

CHOAY Françoise, 1992, Lâallégorie du patrimoine, Le Seuil, Paris.

Clausen Meredith L., 2005, The Pan Am Building and the Shattering of the Modernist Dream, The MIT Press, Cambridge (Mass.) : Londres.

Collins Peter, 1998, Changing Ideals in Modern Architecture, 1750-1950, [1965], seconde édition avec avantpropos de Kenneth Frampton, McGill-Queenôs University Press, Montréal \& Kingston : Londres : Ithaca (traduit en français en 2009, Éditions Parenthèses, Marseille).

CROZIER Michel, FrIEDBERG Erhard, 1977, Lâacteur et le système, Le Seuil, Paris.

Culot Maurice, Lefebvre Philippe, 1980, "Introduction", in André Barey, La Déclaration de Bruxelles. Propos sur la reconstruction de la ville européenne, Archives dôrchitecture moderne, Bruxelles.

EsPAGNE Michel, 1999, Les transferts culturels franco-allemands, Presses universitaires de France, Paris.

GHORRA-Gobin Cynthia, 2006, La théorie du New Urbanism. Perspectives et enjeux, rapport de synthèse, Centre de documentation de lôirbanisme, juillet.

GiedION Sigfried, 1941, Space, Time and Architecture : the Growth of a New Tradition, Harvard University Press, Cambridge (Mass.).

HobSBAwm Eric, RANGER Terence (dir.), 2006, Lôinvention de la tradition, Éditions Amsterdam, Paris, ouvrage traduit de lôAnglais par Christine Vivier, publié en 1983, Syndicate of the Press of the Université of Cambridge.

НгтснСоск Philip Johnson, 1932, The International Style. Architecture since 1922, W. W. Norton \& company, inc., New York.

HiтснСоск Henry-Russell, 1958, Architecture, 19th and 20th Century, Penguin Books, Harmondsworth, Middlesex.

JACOBS Jane, 1961, The Death and Life of Great American Cities, Random House, New York.

JAUSS Hans Robert, 1974, Pour une esthétique de la réception, Gallimard, Paris.

JENCKS Charles, 1985, Le langage de lâarchitecture post-moderne, Denoël, Paris (1 $1^{\text {ère }}$ édition en 1977).

KaTz Peter, 1993, The New Urbanism. Toward an Architecture of Community, Mac Graw Hill, New-York.

King Anthony D., 2000, Globalized Localities or Localized Globalities? Old Wine, New Bottles?, Workshop on The Culture and Politics of Place, Locality and Globalization, Departments of Anthropology and Philosophy, University of California, Santa Cruz, 28 octobre, 
http://www2.ucsc.edu/globalinterns/cpapers/king.pdf.

KoOlHAAS Rem, Harvard project on the city, Stefano Boeri, Multiplicity, Sanford Kwinter, Nadia Tazi, Hans Ulrich Obrist, 2000, Mutations, catalogue dêexposition, Bordeaux, Entrepôt/Arc en Rêve Centre dôrrchitecture, du 24 novembre 2000 au 25 mars 2001, Bordeaux, Arc en rêve Centre d'architecture, ACTAR, Barcelone.

La Conférence dôAthènes sur la conservation artistique et historique des monuments (1931), 2002, édition établie par Françoise Choay, Les Éditions de lâmprimeur, Paris.

LuCAN Jacques, 2001, Architecture en France, 1940-2000, histoire et théories, Éditions du Moniteur, Paris.

LEFEBVRE Henri, 1968, Le droit à la ville, Anthropos, Paris.

LEPETIT Bernard, 1996, "De lấchelle en histoire", in Jacques Revel (dir.), Jeux dấchelles. La micro-analyse à lâxpérience, Gallimard : Seuil, Paris, 71-93.

Migayrou Frédéric et MenNan Zeynep (dir.), 2003, Architectures non standard, catalogue dâexposition, Centre Georges Pompidou, Paris.

MinNAERT Jean-Baptiste (dir.), 2005, Histoires dâarchitectures en Méditerranée XIXe-XX ${ }^{e}$ siècles. Écrire lốistoire dồn héritage bâti, Éditions de la Villette, Paris.

MinNAERT Jean-Baptiste (dir.), 2006, "Nouvelles approches en architecture", Histoire de lâart, n55, octobre.

MinNAERT Jean-Baptiste (dir.), 2009, "Architecture ordinaire et hommes pluriels", in Carmen Popescu (dir.), "Lâutre Europe", Ligeia, n 93-96, juillet-décembre, 38-44.

NASR Joe, VolatT Mercedes (dir.), 2003, Urbanism: Imported or Exported? Native Aspirations and Foreign Plans, actes du colloque de Beyrouth en 1998, Wiley Academy, Chichester.

PeVsner Nikolaus, 1936, Pioneers of the Modern Movement from William Morris to Walter Gropius, Faber \& Faber, Londres.

PICON Antoine, 2010, Culture numérique et architecture. Une introduction, Birkhauser, Bâle.

Prost Antoine, 1996, Douze leçons sur lôhistoire, Le Seuil, Paris.

Risselada Max, VAn Den Heuvel Dirk (dir.), 2005, Team 10, 1953-1981, in Search of a Utopia of the Present, catalogue dêxposition, Netherlands Architecture Institute, Rotterdam.

Rossi Aldo, 1966, Lâarchitettura della Città, Marsilio Editori, Padou.

Sejima Kazuyo (ed.), 2010, People Meet in Architecture, cat. exp. Biennale dôrchitecture de Venise, Marsilio, Venise.

TAFURI Manfredo, 1968, Teoria e storia dellâirchitettura, Laterza, Bari.

TAFURI Manfredo, 1979, Projet et utopie, de lầvant-garde à la métropole, Dunod Paris (édition originale en $1973^{\circ}$.

TAFURI Manfredo, FRANCESCO Dal Co, 1982, Architecture contemporaine, Berger-Levrault, Paris, (édition originale en 1976).

TEXIER Simon, 2005, Paris contemporain. Architecture et urbanisme. De Haussmann à nos jours, une capitale à l'ère des métropoles, Parigramme, Paris.

Venturi Robert, 1966, Complexity and Contradiction in Architecture, The Museum of Modern Art, New York.

WERNER Michael, ZimMERMANn Bénédicte, 2003, "Penser lâhistoire croisée : entre empirie et réflexivité", Annales. Histoire, Sciences sociales, $2003 / 1,58^{\mathrm{e}}$ année.

ZYTNICKI Colette, KAZDAGHLI Habib (dir.), 2009, Le tourisme de lôEmpire français. Politiques, pratiques et imaginaires $\left(X I X^{e}-X X^{e}\right.$ siècles) : un outil de la domination coloniale ?, Publications de la Société française dôhistoire de lộutre-mer Saint-Denis. 